Proc. of 12th International Workshop on Positron and Positronium Chemistry, August 28-September 1, 2017, Lublin, Poland

\title{
Human Tissue Investigations Using PALS Technique Free Radicals Influence
}

\section{B. Jasińska ${ }^{a, *}$, B. ZGardzińska ${ }^{a}$, G. Cholubek ${ }^{b}$, M. Pietrow ${ }^{a}$, M. Gorgol $^{a}$,}

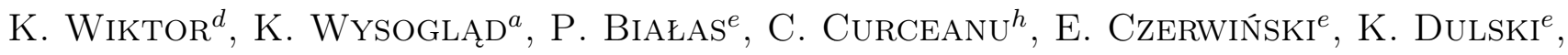
A. Gajos ${ }^{e}$, B. GŁowacz ${ }^{e}$, B.C. Hiesmayr ${ }^{i}$, B. JodŁowska-JędryCh ${ }^{j}$, D. KAmińska ${ }^{e}$,

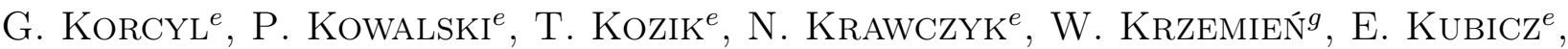
Muhsin Mohammed ${ }^{e, k}$, M. PaWlik-NiedźWiecka ${ }^{e}$, S. NiedźWiecki ${ }^{e}$, M. PaŁka $^{e}$,

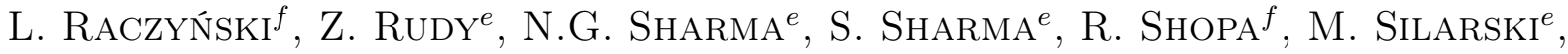
M. Skurzok ${ }^{e}, \mathrm{~A} . \mathrm{WieczoreK}^{e}, \mathrm{H} . \mathrm{WiKtor}^{c}, \mathrm{~W} . \mathrm{Wiślicki}^{f}, \mathrm{M}$. Zieliński ${ }^{e}, \mathrm{P} . \mathrm{Moskal}^{e}$ ${ }^{a}$ Institute of Physics, Maria Curie Skłodowska University, pl. M. Curie Skłodowskiej 1, 20-031 Lublin, Poland

${ }^{b}$ Diagnostic Techniques Unit, Faculty of Nursing and Health Sciences, Medical University of Lublin, Poland ${ }^{c}$ Chair and Department of Gynaecology and Gynaecological Endocrinology, Faculty of Nursing and Health Sciences, Medical University of Lublin, Poland

${ }^{d}$ Department of Obstetrics, Gynaecology and Obstetrics - Gynaecological Nursing,

Faculty of Nursing and Health Sciences, Medical University of Lublin, Poland

${ }^{e}$ Faculty of Physics, Astronomy and Applied Computer Science, Jagiellonian University, 30-348 Cracow, Poland

${ }^{f}$ Department of Complex Systems, National Centre for Nuclear Research, 05-400 Otwock-Świerk, Poland

${ }^{g}$ High Energy Physics Division, National Centre for Nuclear Research, 05-400 Otwock-Świerk, Poland

${ }^{h}$ INFN, Laboratori Nazionali di Frascati, 00044 Frascati, Italy

${ }^{i}$ Faculty of Physics, University of Vienna, 1090 Vienna, Austria

${ }^{j}$ Chair and Department of Histology and Embryology with Experimental Cytology Unit, 20-080 Lublin, Poland

${ }^{k}$ Department of Physics, College of Education for Pure Sciences, University of Mosul, Mosul, Iraq

The positron annihilation lifetime spectroscopy was applied to the samples of the human uterine leiomyomas and the normal myometrium tissues taken from the selected place of the uterus during a surgery. The method indicated differences in values of the measured positron annihilation lifetime spectroscopy parameters (lifetimes and intensities) between healthy and diseased tissue samples. The additional measurements were performed either in darkness or in presence of visible light which influenced the free radicals present in both kind of tissues and, as a result, made changes in free annihilation and $o$-Ps decay lifetime and intensity values.

DOI: 10.12693/APhysPolA.132.1556

PACS/topics: positron annihilation, positronium, free radicals, tissues, uterine leiomyomas

\section{Introduction}

Positron annihilation lifetime spectroscopy (PALS), the method based on a behaviour of thermalized positron in the medium, is commonly used in investigations of various kind of materials: from metals and semiconductors to complex mesoporous substances $[1,2]$.

In the materials where positronium $(\mathrm{Ps})$ is created, the $o$-Ps lifetime correlation with the size of the void in which the positronium is trapped [3-9] allows to determine the void sizes from 0.2 to about $100 \mathrm{~nm}$ and follow many processes undergoing in the medium. PALS techniques were applied, however, in a very limited number of papers concerning living biological systems [10-15]. As the $o$ Ps lifetime reflects changes in the material structure, it

*corresponding author; e-mail: bozena.jasinska@umcs.pl should be connected to the morphology of the cells of the living organisms [16].

A precise understanding of various chemical processes in biological systems is of the greatest importance especially if this understanding contributes to improving a human body diagnosis. The o-Ps and free annihilation lifetime and intensity should be connected to the physiology of these cells. These parameters could be probably used as an indicator of the state of disorder in these cells caused by changes in cells' chemical processes and structure deformations $[16,17]$. A great deal of physiological processes are related to chemical reactions producing or removing free radicals. They influence the processes of positronium formation and positron annihilation and hence could affect the PALS results. The significant difference between a normal and a tumorous tissues is manifested, among others, by different concentrations of free radicals. In general, because tumorous tissues are involved in many unspecific processes and thus 
a greater amount of their genes are involved, so the electron concentration (and a density of other radicals) in altered sample should be larger. They should influence the intensity of free annihilation. The intensity values of PALS components were successfully applied to follow concentration of the free radicals and trapped electrons in the organic materials [18-20]. It has already been shown that illumination of a sample influences the shape of the lifetime spectrum by changing the concentration of free radicals [21-23]. Such results justify an expectation of PALS applicability to free radicals investigations in biological systems.

One of the most known diagnostic techniques, positron emission tomography (PET), is also based on positron behavior in the living organism therefore in principle it is possible to implement results of PALS measurements to future PET diagnostic ability. The innovative construction concept was recently proposed by the J-PET collaboration [24-31]. One of the advantages of J-PET is a possibility of multi-photon imaging [32, 33] which enables the diagnosis based on positron and positronium lifetime [16] as well as on the ratio of $3 \gamma$ and $2 \gamma$ annihilation rates $[17,34,35]$.

In this paper, the healthy and diseased human uterus tissues are investigated using PALS in order to compare modifications in lifetime and intensity values for samples caused by visible light. The uterus was chosen as an object of investigation as it is possible to obtain samples of normal and altered tissues from the same organ of the same patient so the observed changes in PALS parameters are due to the alteration only and are not related to other factors which can differ from patient to patient. Additionally, the presence of oxidative DNA damage and DNA adducts in uterus was stated before [36-40].

\section{Experimental}

The tissues were provided from the patients qualified for a surgical removal of the changed tissue. The samples were taken directly from the organ, just after a removal of the uterus by the surgeon in the operating room. The tissue fragments were taken from the sites altered macroscopically (A) and those identified as a normal (N) tissue. Each sample, with the total volume of about $1.5 \mathrm{~cm}^{3}$, was placed in a cylindrical, steel PALS chamber, divided in half by a Plexiglas partition element and closed with a steal seal. The samples were delivered to the PALS laboratory in transporting thermos, at the constant temperature $\left(23 \pm 1^{\circ} \mathrm{C}\right)$ in less than $1 \mathrm{~h}$. In our laboratory, a ${ }^{22} \mathrm{Na}$ source (of the activity $0.7 \mathrm{MBq}$ ) was placed inside the Plexiglas partition and inserted between two slices of investigated sample. Each sample was measured for $1.5 \mathrm{~h}$ using a standard fast-slow PALS spectrometer. Gamma quanta from positron creation and positron/positronium annihilation were collected by two $\mathrm{BaF}_{2}$ scintillation detectors. The time range of time-to-amplitude converter (TAC) was set on $100 \mathrm{~ns}$, which allowed for a precise measurement of lifetimes up to a few ns. The resolution curve was approximated with the use of a single Gaussian, with the FWHM of about 0.25 ns. The lifetime spectra were analyzed with the use of LT 9.2 [41] program and three components, ascribed to: $p$-Ps, unbound positrons and $o$-Ps annihilation, were distinguished.

After the measurement in the darkness, the sample was transferred to a quartz glass container and then the spectra for the illuminated sample were collected (light from a $20 \mathrm{~W}$ lamp placed at the distance of $40 \mathrm{~cm}$, to minimize the sample heating). After PALS measurements, the samples were thoroughly rinsed with formalin and transported to the hospital for a histopathological examination. The histopathological photos clearly show the morphological diversity of the tested samples [15].

Our study was made under the approval of the Bioethics Committee of the Medical University of Lublin.

\section{Results and discussion}

From PALS measurement it was found that there exist significant differences of results obtained from different female patients as well as between normal $(\mathrm{N})$ and altered (A) tissues from the same patient [15]. Comparison of results for the first points in regions II and III in Fig. 1 confirms this conclusion.
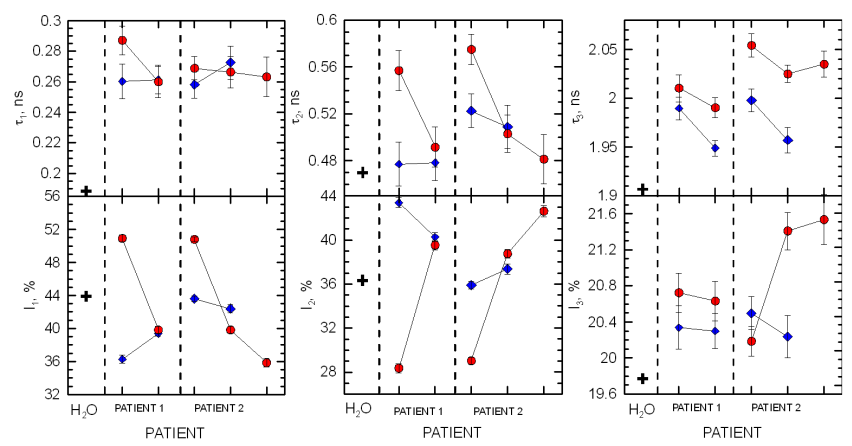

Fig. 1. The lifetime and intensity values for normal (blue diamonds) and altered (red dots) uterine tissue. Upper and lower parts show the mean lifetime and intensity of three main annihilation components originating from para-positronium (left), direct annihilation (middle) and ortho-positronium (right). The first points for both patients were measured in darkness, the second (and third - elongated exposition time) - light illuminated. Three regions in the figure from left to right: deionized water at room temperature (crosses), patient I, patient II.

Preliminary measurements of free radicals examination presented here were performed for two patients from the same group of samples. In Fig. 1 results of measurements for both patients in darkness and exposed to the light are shown. From PALS parameter one can conclude that an exposition of the samples to light makes a significant impact on altered tissue only (Fig. 1, red points). The greatest difference can be seen for free positrons (second component). The lifetime $\tau_{2}$ is significantly shortened for the illuminated altered tissue samples, while the adequate intensity fraction increases. In the case of normal tissue samples, no such effects are observed during illu- 
mination of samples - the differences in the PALS parameters in illuminated and non-illuminated samples are small. In the tissues positron can interact with both the dense matter and the biofluids. To estimate the influence of processes related to water (in cells), lifetime and intensity values of all components determined in deionized water are also presented for comparison (crosses).

A higher density of electrons (radicals) in the altered sample results in increasing probability of the free $\mathrm{e}^{+}$ annihilation and the probability of the positronium creation.

\section{Conclusions}

Light influences significantly results of the PALS measurements obtained for cancerous samples while this effect for normal tissues is negligible or barely marked for $o$-Ps lifetime value only. It should be noted that the metabolism of dying cells may additionally affect the results of the measurements as well as the diversity in physiological processes of patients. However, the presented results of these pilot studies are encouraging for applying PALS and PET in early stages of pathogenic alteration in the body.

\section{Acknowledgments}

B.C. Hiesmayr gratefully acknowledges the Austrian Science Fund FWF-P26783.

\section{References}

[1] D.M. Schrader, Y.C. Jean, Positron and Positronium Chemistry, Eds. D.M. Schrader, Y.C. Jean, Elsevier, Amsterdam 1988.

[2] P.G. Coleman, Positron Beams and Their Applications, Ed. P.G. Coleman, World Sci., Singapore 2000.

[3] S.J. Tao, J. Chem. Phys. 56, 5499 (1972).

[4] M. Eldrup, D. Lightbody, J.N. Sherwood, Chem. Phys. 63, 51 (1981).

[5] B. Jasińska, A.E. Kozioł, T. Goworek, J. Radioanal. Chem. 210-211, 617 (1996).

[6] B. Jasińska, A.E. Kozioł, T. Goworek, Acta Phys. Pol. A 95, 557 (1999).

[7] T. Goworek, K. Ciesielski, B. Jasińska, J. Wawryszczuk, Chem. Phys. Lett. 272, 91 (1997).

[8] T. Goworek, K. Ciesielski, B. Jasińska, J. Wawryszczuk, Chem. Phys. 230, 305 (1998).

[9] D.W. Gidley, W.E. Frieze, T.L. Dull, A.F. Yee, E.T. Ryan, H.-M. Ho, Phys. Rev. B 60, R5157 (1999).

[10] Y.C. Jean, H.J. Ache, J. Am. Chem. Soc. 99, 1623 (1977).

[11] G. Liu, H. Chen, L. Chakka, J.E. Gadzia, Y.C. Jean, Phys. Status Solidi C 10, 3912 (2007).

[12] Y.C. Jean et al., Appl. Surf. Sci. 252, 3166 (2006).

[13] E. Axpe et al., PLoS One 2, 83838 (2014).
[14] E. Kubicz et al., Nukleonika 60, 749 (2015)

[15] B. Jasinska et al., Acta Phys. Pol. B 48, 1737 (2017).

[16] P. Moskal et al., Patent Application: P 405185 (2013); PCT/EP2014/068374 (2014); WO2015028604.

[17] B. Jasińska, P. Moskal, Patent Application: P418689 (2016); PCT/IB2017/055560.

[18] M. Pietrow, J. Wawryszczuk, Mater. Sci. Forum 666 89 (2011).

[19] R. Zaleski, B. Zgardzińska, M. Pietrow, T. Goworek, Rad. Phys. Chem. 77, 1306 (2008).

[20] M. Pietrow, B. Zgardzińska, Acta Phys. Pol. A 110, 641 (2006).

[21] B. Zgardzińska, T. Goworek, Acta Phys. Pol. A 119 , 238 (2011).

[22] T. Goworek, M. Pietrow, R. Zaleski, B. Zgardzinska, Chem. Phys. 355, 123 (2009).

[23] B. Zgardzińska, T. Hirade, T. Goworek, Chem. Phys. Lett. 446, 309 (2009).

[24] P. Moskal et al., Nucl. Instrum. Methods Phys. Res. A 764, 317 (2014)

[25] L. Raczynski et al., Nucl. Instrum. Methods Phys. Res. A 764, 186 (2014).

[26] P. Moskal et al., Nucl. Instrum. Methods Phys. Res. A 775, 54 (2015).

[27] L. Raczynski et al., Nucl. Instrum. Methods Phys. Res. A 786, 105 (2015).

[28] G. Korcyl et al., Acta Phys. Pol. B 47, 491 (2016).

[29] P. Moskal et al., Phys. Med. Biol. 61, 2025 (2016).

[30] L. Raczynski et al., Phys. Med. Biol. 62, 5076 (2017).

[31] J. Smyrski et al., Nucl. Instrum. Methods Phys. Res. A 851, 39 (2017).

[32] A. Gajos et al., Nucl. Instrum. Methods Phys. Res. A 819, 54 (2016).

[33] D. Kamińska et al., Eur. Phys. J. C 76, 445 (2016).

[34] B. Jasińska, P. Moskal, Acta Phys. Pol. B 48, 1577 (2017).

[35] B. Jasińska, B. Zgardzińska, Acta Phys. Pol. A 132, 1616 (2017)

[36] J.D. Yager, Steroids 99A, 56 (2015).

[37] M. Sharma, D.E. Shubert, M. Sharma, K.J. Rodabaugh, B.P. McGarrigle, C.M. Vezina, D.P. Bofinger, J.R. Olson, Biochem. Biophys. Res. Commun. 307, 157 (2003)

[38] H. Wiktor, M. Kankofer, I. Schmerold, A. Dadak, M. Łopucki, H. Niedermuller, Virchows Arch. 445 , 74 (2004)

[39] H. Wiktor, M. Kankofer, H. Źrubek, M. Podolak, Prenat. Neonat. Med. 3, 81 (1998).

[40] H. Wiktor, G. Buszewicz, P. Kozioł, M. Sikorski, R. Madro, H. Źrubek, in: Proc. Balkan Congress of Oncology, Athens, 1996, p. 569.

[41] J. Kansy, Nucl. Instrum. Methods Phys. Res. A 374, 235 (1996). 